\title{
A Novel State-of-charge Estimation Method of Lithium-ion Batteries Combining the Grey Model and Genetic Algorithms
}

\author{
Lin Chen, Zhengzheng Wang, Zhiqiang Lü, Junzi Li, Bing Ji, Member, IEEE, Haiyan Wei and Haihong \\ Pan
}

\begin{abstract}
In order to guarantee safe and reliable operation of electric vehicle batteries and to optimise their energy and capacity utilisation, it is indispensable to estimate their state-of-charge (SoC). This study aimed to develop a novel estimation approach based on the Grey model and Genetic Algorithms without the need of a high-fidelity battery model demanding high computation power. A SoC analytical model was established using the Grey System theory based on a limited amount of incomplete data in contrast to conventional methods. The model was further improved by applying a sliding window mechanism to adjust the model parameters according to the evolving operating status and conditions. In addition, the Genetic Algorithms were introduced to identify the optimal adjustment coefficient, $\lambda$, in a traditional Grey model $(1,1)$ model to further improve the source estimation accuracy. For experimental verification, two types of Lithium-ion batteries were used as the device-under-test that underwent typical passenger car driving cycles. The proposed SoC estimation method were verified under diverse battery discharging conditions and it demonstrated superior accuracy and repeatability compared to the benchmarking GM method.
\end{abstract}

Index Terms - Electric vehicles (EVs), genetic algorithms, grey model, lithium-ion battery, state-of-charge.

\section{INTRODUCTION}

With a high demand of superior electrochemical energy storage, lithium-ion battery (LIB) is widely used as a primary power source, thanks to its advantages of high energy density,

Manuscript received May 14, 2017; revised July 26, 2017; accepted November 27, 2017. This work was supported in part by the National Natural Science Foundation of China under Grant No.51267002 and Grant No.51667006, in part by Guangxi Natural Science Foundation under Grant No.2015GXNSFAA139287, in part by Innovation Project of Guangxi Graduate Education under Grant No.YCSW2017038, and inpart by Guangxi Key Laboratory of Manufacturing System \& Advanced Manufacturing Technology under Grant No.15-140-30S002. Recommended for publication by Associate Editor M. Ferdowsi. (Corresponding author: Lin Chen.)

L. Chen, Z. Wang, Z. Lü, J. Li, H. Wei and H. Pan are with the Department of Mechatronics Engineering, College of Mechanical Engineering, Guangxi University, Nanning, 530004, China (e-mail: gxdxcl@163.com; wzz5580@163.com; bushlv@hotmail.com; ljzxiangshang@163.com; gxwhytu@163.com; hustphh@163.com).

B. Ji is with the Department of Engineering, University of Leicester, Leicester, LE1 7RH, UK (e-mail: bing.ji@leicester.ac.uk). lightweight, long cyclic lifetime, low self-discharge rate, and almost zero memory effect [1]-[3]. Particularly, electric vehicles (EVs) such as the popular plug-in hybrid EVs (PHEVs) and plug-in EVs (PEVs) will require a high-performance LIB pack to become fully viable options. The battery pack is scalable in capacity and output power by using a collection of cells or module assemblies to meet different energy targets. Its operational dynamics and lifetime are largely dependent on its constituent parts and the cell imbalance among many LIBs accelerates degradation, whilst causing performance deterioration, overheating, and over-charge/discharge leading to bad customer experience and potential fire hazard. Therefore, a battery management system (BMS) is widely used on-board in EV applications; this is indispensable to carry out essential management and diagnostic functions for better performance and reliable operations [4].

\section{A. Review of estimation approaches}

The state-of-charge (SoC) for evaluating the residual capacity of LIBs is a primarily monitored parameter of a BMS and the online estimation of $\mathrm{SoC}$ is just as the fuel gauge function that resides in an internal combustion engine (ICE) vehicle [5]-[8]. Generally, SoC is defined as the ratio of the remaining capacity to the nominal capacity of the battery in percentage. A precise estimation of $\mathrm{SoC}$ is critical which enables maximum utilization of the energy and power of LIBs without adverse effect on their lifetime and avoids over-charge/discharge [7]. Many methods are currently used to calculate the SoC. The Coulomb counting method based on the integral of charging/discharging current is considered as a fundamental and widely used method to estimate the $\mathrm{SoC}$ [9],[10]. However, this method requires the initial $\mathrm{SoC}$ of the battery which is not always available and suffers from the accumulated measurement errors due to noise and drift. Alternatively, an open-circuit voltage (OCV) method can be used based on the correlation between OCV and SoC. However, the OCV measurement is normally intrusive with respect to continuous operating conditions and can only be implemented under specific conditions in practice when the EV is stationary. Moreover, to achieve a high fidelity for a $\mathrm{SoC}$ measurement, a significant blank time should be set, because a short OCV increase is imposed after the current interruption followed by a slow decay [11]. To overcome the drawbacks of the above methods, several battery model-based methods combined with various state estimation techniques have been proposed. An ECM is comprised of capacitors and resistors, which mimics the battery dynamic voltage/current responses, 
while ignoring the physical and chemical processes within the battery cell. ECM, however, tends to suffer from inaccuracy when cells are subject to intense physical and chemical effects, such as under the high charge/discharge rate. Additionally, it is difficult to identify model parameters under different operating conditions. On the other hand, a physics-based electrochemical model is established based on internal cell reactions regarding mass transfers, chemical thermodynamics, and electrodynamics. In this case, many model parameters are required to be determined from the internal material properties of a battery, which requires in-depth cell knowledge and can hardly derived from terminal electrical properties. Moreover, the demand for a high computation power beyond the capacity of microcomputers mandates its suitability for the offline battery performance analysis and cell design only. By adding an adaptive filter for signal processing, the accuracy of SoC estimation can be significantly improved, and one of the commonly used methods is the extended Kalman filter (EKF). The method depends more strongly on the accuracy of model parameters, which are difficult to achieve and computationally intensive. In addition, an inaccurate matrix of the system noise, such as the relevance, mean value and covariance matrix, could lead to filter divergence and affect its stability [12]. To improve the robustness of the EKF, a sigma-point KF (SPKF) is employed to better address the model nonlinearities [13]. And the different forms of this filter including the central difference $\mathrm{KF}$ (CDKF) and unscented KF (UKF) have been proposed to improve the accuracy for SoC estimation [14]. Because it is difficult to obtain a satisfactory tradeoff between fidelity and complexity of a battery model relying on a deterministic electrical model, an artificial intelligence method (also termed as the data-driven method) has been used. A so-called neural network (NN) was proposed in [15], which has demonstrated a high accuracy and robustness owing to its good performance in solving complex nonlinear system problems. The advantage of the NN-based model is that the exact knowledge about the battery behavior is not required. However, a large amount of historical data is required to train the NN. Furthermore, a new battery trained by NN is inaccurate for the SoC estimation of an aged battery because of the open-loop nature of this approach [16]. Support vector machines (SVMs) are also used in SoC estimation [17], [18], which also needs extensive training data and shares similar disadvantages as the NN.

Several automatic control theorems have been used in battery SoC estimation, such as a proportional integral (PI) observer, to overcome the ageing and condition related parametric drift and to improve the accuracy and speed tradeoff in the $\mathrm{SoC}$ estimation. To prepare nonlinear battery models as well as model parameters, researchers proposed an adaptive geometric observer for simplicity. This method was robust in processing uncertainties, and the error dynamics of state and parameter estimation were convergent [19]. To assure the stability of an adaptive observer, Lyapunov theory has been proposed to estimate the $\mathrm{SoC}$ with the advantages of no need for a priori knowledge of the model parameters [20].

\section{B. Contributions of the study}

Most of the recent studies mainly have two shortcomings on SoC estimation. Firstly, these methods demand a large amount of training data. Secondly, a complex and accurately-parameterized battery model is required for accurate estimation of SoC. A Grey model (GM) was introduced to overcome the above-mentioned limitations of conventional methods. GM is a well-known dynamic modeling tool to deal with systems of uncertainty, especially for multivariables, discrete, and incomplete data, which was first proposed by Prof J. Deng in 1982. The superiority of GMs over conventional statistical models manifests in the fact that only a limited amount of non-negative data is needed to predict the systems' behaviors without mathematical models. Grey forecasting models have been widely used in various problems such as foreign currency exchange rates [21], environmental pollution [22], power load [23], policy effectiveness [24], unemployment rates [25], and energy consumption [26]. Furthermore, the sliding mechanism was applied to improve the predicting accuracy of the original GM by updating the input data, and an optimization algorithm was used to optimize the adjustment coefficients of GMs during the sliding process [27].

A key contribution of this study is that the GM was applied to estimate battery SoC, combined with a sliding window GM and Genetic Algorithms (GA), in order to build a sliding GAGM (SGAGM) to further improve the accuracy of SoC estimation. First, the GM $(1,1)$ demands a reduced amount of data and avoids a complex battery model for SoC estimation. Further, to track the data fluctuation dynamically and enhance the precision of estimation, a sliding window mechanism was introduced to update the forecasting sequences for estimation. Finally, the GA, a global optimization technique, was used to estimate and update the adjustment coefficient $\lambda$ of GM $(1,1)$ model at each window to further improve the estimation accuracy. A series of experiments were conducted to evaluate the performance of SGAGM. The prediction accuracy was justified by quantitative comparisons using a group of evaluation criterion such as the mean relative error (MRE), post-error ratio, and degree of Grey relation.

\section{Organization of the paper}

The paper is organized as follows: In section II, the GM (1, 1) and SGAGM are discussed as well as the evaluation of their prediction accuracy. Section III provides the experimental design, test rig and experiment conditions. The results and discussion are presented in Section IV followed by the conclusions in Section V along with the future work.

\section{Methodology}

GM $(1,1)$ is a most widely used GM to deal with systems with uncertain and imperfect information. By determining the intrinsic trend of data changes, the $\operatorname{GM}(1,1)$ can provide sufficient accurate prediction with a minimal amount of data.

\section{A. $\operatorname{GM}(1,1)$}

GM $(1,1)$ is a time-series forecasting model; this model is most frequently applied for predictions due to its simplicity. The GM $(1,1)$ procedure is as follows [28, 29]:

Step 1: The non-negative historical sequence $X^{(0)}$ is expressed as follows:

$$
X^{(0)}=\left\{x_{(k)}^{(0)}\right\}_{k=1}^{n}
$$

Step 2: When this sequence is subjected to Accumulating 
Generation Operation (1-AGO), the following sequence $x_{(k)}^{(1)}$ is obtained. The AGO is expressed as follows:

$$
X^{(1)}=\left\{x_{(k)}^{(1)}\right\}_{k=1}^{n}
$$

where,

$$
x_{(k)}^{(1)}=\sum_{i=1}^{k} x_{(i)}^{(0)}, k=1,2, \cdots, n
$$

Step 3: The whitening grey dynamic model can be acquired using $a$ first-order differential equation with constant coefficient $b$,

$$
\frac{d x_{(k)}^{(1)}}{d k}+a x_{(k)}^{(1)}=b
$$

where $a$ is the development coefficient and $b$ is the influence of the external impact. The $\hat{a}=[a, b]^{T}$ is a sequence of parameters that can be obtained as follows:

$$
\hat{\mathbf{a}}=\left(\mathbf{B}^{T} \times \mathbf{B}\right){ }^{1} \times \mathbf{B}^{T} \times \mathbf{Y}_{N}
$$

where

$$
\mathbf{B}=\left[\begin{array}{cc}
-0.5 \times\left(x_{(2)}^{(1)}+x_{(1)}^{(1)}\right) & 1 \\
-0.5 \times\left(x_{(3)}^{(1)}+x_{(2)}^{(1)}\right) & 1 \\
\vdots & \vdots \\
-0.5 \times\left(x_{(n)}^{(1)}+x_{(n-1)}^{(1)}\right) & 1
\end{array}\right]
$$

and

$$
\mathbf{Y}_{N}=\left[x_{0}(2) \quad x_{0}(3), \cdots, x_{0}(n)\right]^{T}
$$

Using Eq. (4), the solution of the whitening differential equation is obtained,

$$
\hat{x}_{(k+1)}^{(1)}=\left(x_{(1)}^{(0)}-\frac{b}{a}\right) \times e^{-a k}+\frac{b}{a}, k=1,2, \cdots, n-1
$$

To obtain the forecasting values of $\hat{x}_{(k)}^{(0)}$, the inverse accumulated generating operation (IAGO) is used to establish the following GM (9),

$$
\hat{x}_{(\mathrm{k}+1)}^{(0)}=\hat{x}_{(\mathrm{k}+1)}^{(1)}-\hat{x}_{(\mathrm{k})}^{(1)}, k=0,1,2, \cdots, n-1
$$

where, $\hat{x}_{(1)}^{(0)}=\mathrm{x}_{(1)}^{(0)}$.

The detailed implementation schematic of $\operatorname{GM}(1,1)$ is shown in Fig. 1:

Fig. 1. Implementation schematic of GM $(1,1)$

\section{B. SGAGM}

The traditional GM $(1,1)$ model has many advantages, but inevitable limitations as well. The prediction relies on the system comprising of an exponential function, and the model parameters cannot be adjusted according to system changes. However, in application, the trend of data generally changes over time, making the data to exhibit different characteristics. The whitening process of the traditional GM $(1,1)$ grey differential equation is solved by converting the differential into a differential form. In addition, the different whitening processes will obtain different whitening equations, the final development coefficient, and the amount of grey. Thus, improvements can be made by updating model parameters according to the latest information and by introducing an adjustment coefficient $\lambda$ into the traditional GM $(1,1)$. To update the model parameters according to the latest information, the sliding mechanism is the best choice as it can dynamically use the newest data to establish a model in each window. Because of the implicit parallelism and intelligence, GAs are ideally suited to search for the best (globally optimized) solution from a large multidimensional solution space [30]. Therefore, the GAs are used to optimally determine the adjustment coefficient value of $\lambda$.

The frame of SGAGM is divided into three stages:

Stage 1 - with an adjustment coefficient $\lambda$ for GM (1, 1, ג):

Because the GM $(1,1)$ is whitened using the grey equation, the differential equation is solved by the difference in the form of (4). However, the differential difference equations are divided into the forward and backward difference such as (10) and (11), it is not necessarily consistent with the real situation of the system only through the simple average effect.

$$
\begin{aligned}
& \frac{x_{1}\left(t_{1}\right)-x_{1}\left(t_{2}\right)}{t_{1}-t_{2}}+a x_{1}\left(t_{1}\right)=b \\
& \frac{x_{1}\left(t_{1}\right)-x_{1}\left(t_{2}\right)}{t_{1}-t_{2}}+a x_{1}\left(t_{2}\right)=b
\end{aligned}
$$

Therefore, (10) and (11) were weighted by the weighting factor $\lambda$ for correcting the prediction result of $\operatorname{GM}(1,1), \lambda \in$ $[0,1]$, and the weighting process is shown in (12).

$$
\frac{x_{1}\left(t_{1}\right)-x_{1}\left(t_{2}\right)}{t_{1}-t_{2}}+a\left[\lambda \times x_{1}\left(t_{1}\right)+(1-\lambda) \times x_{1}\left(t_{2}\right)\right]=b
$$

Where $\hat{\mathbf{a}}=[a, b]^{T}$ is determined using (5). However, $\mathbf{B}$ is shown in (13), and the $Y_{N}$ is the same as (7).

$$
\mathbf{B}=\left[\begin{array}{cc}
-\lambda \times x_{(1)}^{(1)}-(1-\lambda) \times x_{(2)}^{(1)} & 1 \\
-\lambda \times x_{(2)}^{(1)}-(1-\lambda) \times x_{(3)}^{(1)} & 1 \\
\vdots & \vdots \\
-\lambda \times x_{(n-1)}^{(1)}-(1-\lambda) \times x_{(n)}^{(1)} & 1
\end{array}\right]
$$

Using (8) and (9), the solution is solved. Thus, the value of $\hat{x}_{(k)}^{(0)}$ is finally determined by $\lambda$.

\section{Stage 2 - Optimization of $\lambda$ by GAs:}

The GAs are used to calculate and acquire the optimal value of $\lambda$, thus the GM optimized by GA (GAGM) was acquired. GAGM provides an adjustment coefficient $\lambda$ according to the differential form of differential, which makes the traditional GM $(1,1)$ achieve adjustable parameters and improve the lack of flexibility of $\operatorname{GM}(1,1)$ in the traditional mean form. The scope of application for $\operatorname{GM}(1,1)$ is extended to $\left(\frac{1}{\lambda}, \frac{1}{1-\lambda}\right)$. The principle of a GA-based optimization technique is briefly highlighted below:

(1) Individual Coding: Binary coding. The $\lambda, \lambda \in[0,1]$, is divided by a group $n$-bit binary string to build different individuals. Individual precision can be controlled by selecting different $n$. In this study, we selected $n=20$, and the accuracy of each individual $\lambda$ is 6 decimals.

(2) Initialization: Generation of a large number, $K$, of 
prospective solutions, usually randomly. In this study, we selected $K=20$.

(3) Fitness Function: Fitness Function of the deviation of each solution (fitness test) based on the objective function (goal). In this study, the Fitness Function is shown as (14).

$$
\operatorname{Fit}\left(\lambda_{i}\right)=n / \sum_{t=1}^{n}\left|\frac{x_{0}(t)-\hat{x}_{0}(t)}{x_{0}(t)}\right|
$$

(4) Selection: Selection of the best solution and passing their "genes" to the following generations, followed by rejection of the rest. In this study, we selected the best gene by Roulette and passed the gene to a new generation.

(5) Crossover: Random selection of pairs of the best solution (parent chromosome) and their exchanging to produce new children (i.e., solutions) that inherit genes from the parent individuals. Here, chromosome refers to the initial population composed of many solutions to the problem. This process is shown in (15) and (16). Moreover, the crossover rate is set as $p_{c}$ $=0.8$.

$$
\begin{aligned}
& X^{\prime}=r X+(1-r) Y \\
& X^{\prime}=(1-r) X+r Y
\end{aligned}
$$

Where, $r=U(0,1)$.

(6) Mutation: This is an operator that typically works with a low probability to reintroduce lost genes into the population. In this study, the mutation rate was set as $p_{m}=0.5$.

(7) Evolution: After ranking all the individuals, those with a high fitness remain and those with a low fitness were removed.

(8) Stop: Continuation of steps (2)-(7) until a population of the best solutions was achieved.

\section{Stage 3 - Sliding mechanism for GAGM:}

To clearly describe the Grey theory with sliding mechanism, the sliding GAGM $(1,1)$ model is used for illustration. Here, we assume that simplify GAGM $(1,1)$ uses $M$ number of data points $(M \geq 4)$ to predict $N$ number of data points $(N \geq 1)$.

The steps of sliding $\operatorname{GAGM}(1,1)$ are described as follows:

Step 1: for instance, beginning with $x_{(1)}^{(0)}$, creating GAGM $(1, \quad 1)$ by using data $\left\{x_{(1)}^{(0)}, x_{(2)}^{(0)}, \cdots, x_{(k)}^{(0)}\right\}$; then, data $\left\{\hat{x}_{(k+1)}^{(0)}, \hat{x}_{(k+2)}^{(0)}, \cdots, \hat{x}_{(k+N)}^{(0)}\right\}$ will be predicted. This can be shown as follows:

$$
\left.\left.\operatorname{GAGM}(1,1)\left\{x_{(k)}^{(0)}\right)\right\}_{k=1}^{M} \rightarrow\left\{x_{(k)}^{(0)}\right)\right\}_{k=M+1}^{M+N}
$$

Step 2: To utilize new data for prediction. Therefore, in this step, $\operatorname{GAGM}(1,1)$ will be remodeled when $m$ new data points are acquired. For example, when new data $\left\{x_{(k)}^{(0)}\right\}_{k=M+1}^{M+m}$ are obtained, the previous data $\left\{x_{(k)}^{(0)}\right\}_{k=1}^{m}$ should be removed, and a new GAGM $(1,1)$ model is established using the series $\left\{x_{(k)}^{(0)}\right\}_{k=m+1}^{M+m}$. Later, by using the new model, the series $\left\{x_{(k)}(0)\right\}_{k=M+m+1}^{M+m+N}$ is predicted. This step can be illustrated as follows:

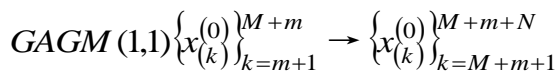

Step 3: Steps 1 and 2 should be iteratively implemented until the execution for the last data point is completed.

In this paper, we propose a SoC estimation method by using an efficient technique of sliding mechanism to increase the accuracy in the forecasting of GAGM. The initial SoC was corrected using the OCV method, providing the OCV-SoC-temperature relationship offline. Then, the discharge capacity was acquired online using the Coulomb counting method. Finally, the SGAGM was used for SoC estimation based on the historical $\mathrm{SoC}$, which was calculated from the historical discharge capacity. The implementation schematic of the SGAGM for SoC estimation is shown in Fig. 2. We used the SGAGM (M, $m, N)$ to express the combination of sliding mechanism with the GAGM. The $M$ represents the number of window size $(M \geq 4), m$ represents the number of sliding data size $(m \geq 1)$, and $N$ represents the number of predicted data size $(N \geq 1)$.

Fig. 2. Implementation schematic of SGAGM for SoC estimation

\section{Evaluation of predicted accuracy}

Usually, the mean relative error $\alpha$ (MRE), degree of grey relation $\gamma$, and post-error ratio $C$ are used to test and determine the accuracy of grey prediction model. The $\gamma$ is determined using Grey relational analysis (GRA) [31].

In the original sequence $X^{(0)}$ and its predicted equivalent $\hat{X}^{(0)}$, the forecasting errors between the two sequences are $\varepsilon^{(0)}$. The mean values and covariance for both the forecasted sequence and forecasting errors $\alpha, \bar{x}, S_{1}, \bar{\varepsilon}$, and $S_{2}$ are:

$$
\begin{gathered}
\alpha=\frac{1}{n} \sum_{k=1}^{n}\left|\varepsilon_{(k)}^{(0)}\right| \\
\bar{x}=\frac{1}{n} \sum_{k=1}^{n} \mathbf{x}_{(k)}^{(0)} \\
S_{1}=\sqrt{\frac{1}{n-1} \sum_{k=1}^{n}\left(x_{(k)}^{(0)}-\bar{x}\right)^{2}} \\
\bar{\varepsilon}=\frac{1}{n} \sum_{k=1}^{n} \mathbf{\varepsilon}_{(k)}^{(0)} \\
S_{2}=\sqrt{\frac{1}{n-1} \sum_{k=1}^{n}\left(\varepsilon_{(k)}^{(0)}-\bar{\varepsilon}\right)^{2}}
\end{gathered}
$$

The post-error ratio $C$ is shown in (24).

$$
C=\frac{S_{2}}{S_{1}}
$$

MRE denotes the prediction accuracy. The smaller the MRE, the higher the accuracy of prediction. The post-error ratio $C$ indicates the change rate of the forecasting error. The smaller the $C$ value, the better the forecasting accuracy. The $\gamma$ represents the similarity degree of the raw and predicted data, which reflect the accuracy from another aspect, and the high the $\gamma$, the superior the forecasting accuracy.

\section{EXPERIMENTAL}

\section{A. Construction of test bench for LIBs}

The experiment data originated from two types of LIBs and can be divided into two parts: the constant current (CC) tests 
and dynamic tests. The $\mathrm{CC}$ test data were obtained from NASA's data sets [32], and the dynamic test data were obtained from a test bench. This consists of an environmental chamber, ITECH company's electronic load instrument IT8511A+, ITECH company's IT6523D as a charger, a suite of sensors (voltage, current, and temperature), customized switching relays, data acquisition system (DAQ card), and a PC for control and analysis.

The major equipment parameters of IT8511A+ and ITECH IT6523D are listed in Table I.

TABLE I

MAJOR EQUIPMENT PARAMETERS

The configuration of PC: CPU: Intel(R) Core(TM) I7-2670QM CPU @ 2.20GHz; RAM: 8GB. The PC runs on Microsoft Windows 7 operating system, and the required software package is MATLAB 2014a. The battery data including the terminal voltage, load current, discharge capacity (Ah), temperature, and other signals can be collected and recorded during the charging and discharging. The data acquisition frequency was $1 \mathrm{~Hz}$, and the recorded data (terminal voltage, load current, and discharged capacity) were transmitted to the control computer via a USB cable. The proposed method was added to the control computer using LabVIEW to achieve online SoC estimation. Furthermore, if the battery parameters such as the terminal voltage, load current, or temperature exceeded the normal values, the control computer would open the circuit to stop charging or discharging to achieve circuit protection. The physical experimental platform is shown in Fig. 3.

Fig. 3. Physical experimental platform: (a) charging process of battery, (b) discharging process of battery

TABLE II

A COMPARISON OF THE SPECIFICATIONS OF TWO TYPES OF BATTERIES

To test the predictive performance of SGAGM under different conditions, two types of batteries were selected for testing. A comparison of the specifications of both types of batteries is shown in Table II. The battery selected from NASA's battery (18650 Gen 2 high-power) was used at three different ambient temperatures: $4{ }^{\circ} \mathrm{C}, 24{ }^{\circ} \mathrm{C}$, and $44{ }^{\circ} \mathrm{C}$. In each set of tests, LIBs were charged in the $\mathrm{CC}$ mode at $1.5 \mathrm{~A}$ until the battery voltage reached the top cut-off voltage (set as $4.2 \mathrm{~V}$ ); then, they were charged in the constant voltage $(\mathrm{CV})$ mode until the charging current dropped below $20 \mathrm{~mA}$. Later, discharging is performed from $4.2 \mathrm{~V}$ to different cut off voltages (COV), such as $2.0 \mathrm{~V}$ and $2.7 \mathrm{~V}$, in CC mode at a level of two different discharge currents (2A, 4A). The dynamic test data come from LIB (Samsung ICR18650) cells. The LIBs were charged in CC mode until a top cut-off voltage was reached (here $4.2 \mathrm{~V}$ ), and then they were charged in the $\mathrm{CV}$ mode until the charging current dropped below $26 \mathrm{~mA}$. The experiment temperature was set at $25{ }^{\circ} \mathrm{C}$. Moreover, it subjected to discharge at different temperatures and load conditions using a controlled power source, electronic loads, and environmental chamber.

\section{B. Battery Testing}

SGAGM is equivalent to adding a new information perturbation to increase the weight of new information. To test the predictive performance of SGAGM at different temperatures and load conditions, experiments were designed as follows: (1) Discharge was carried out at different $\mathrm{CC}$ levels of $2 \mathrm{~A}$ and $4 \mathrm{~A}$ at different temperatures $\left(4^{\circ} \mathrm{C}, 24{ }^{\circ} \mathrm{C}\right.$, and 44 ${ }^{\circ} \mathrm{C}$ ), and the corresponding cycles were as follows: cycle 290 of B0005, cycle 46 of B0029, cycle 241 of B0033, and cycle 69 of B0053 (from NASA). (2) Discharge was carried out at a dynamic condition - New European Driving Cycle (NEDC) and Japanese 10.15 Model Driving Schedule (JP1015) - at a temperature of $24{ }^{\circ} \mathrm{C}$ for battery \#11. The test results of dynamic condition are shown in Fig. 4. The specific experiments for different batteries are shown in Table III.

Fig. 4. Measured cell response in the dynamic condition test. (a) New European Driving Cycle. (b) Japanese 10.15 Model Driving Schedule

TABLE III

LIST OF EXPERIMENTAL BATTERIES

\section{RESULTS AND DISCUSSION}

In this section, GM $(1,1)$, SGAGM and NN methods were compared for SoC estimation. The window size of SGAGM was set as $M=8$. The sliding size was set to $m=1$. Further, the predicted data size was set as $\mathrm{N}=1$. The GM $(1,1)$ used top $10 \%$ of the original data to predict the remaining $90 \%$ of the data. For the NN method to estimate SoC, characteristic parameters of battery such as battery terminal voltage, discharge current, and battery temperature were selected as the inputs of the NN, and battery SoC was the output.

\section{A. Comparison of SoC estimation results}

The experimental results of battery discharging under different conditions are shown in Figs. 5 and 6.

To verify the adaptability of the model to the actual working conditions, the experimental results were achieved under different temperatures and different discharging conditions. The SGAGM does not depend on a well-parameterized battery model; therefore, it can achieve a higher estimation accuracy on both the $\mathrm{CC}$ and dynamic loading profile than that of the model-based methods, which cannot be applied to parameter identification on CC. To justify its superiority over common methods, a traditional $\mathrm{NN}$ was used to compare with the SGAGM to validate the accuracy of SoC estimation. Figs. 5 (a)-(d) show the prediction curve of NASA battery (18650 Gen 2 high power) at constant discharge magnification and different temperatures; the prediction curve using $\mathrm{GM}(1,1)$ and reference curve are basically the same at the first $500 \mathrm{~s}$. However, the forecast values significantly deviated from the $\mathrm{SoC}$ reference after $500 \mathrm{~s}$. The NN is relatively accurate for tracking the changes in SoC on the overall trend. However, the prediction curves using NN still have a large local fluctuation. The prediction curves using SGAGM and the reference curves are basically the same throughout the forecast period. Figs. 5 (e)-(f) show the prediction curve of LIB (Samsung ICR18650) cells at different dynamic conditions and the same temperatures. Similarly, the prediction curve using GM $(1,1)$ and reference curve are basically the same at the first $1000 \mathrm{~s}$. However, the predicted effect is too poor to be accepted after $1000 \mathrm{~s}$. The local fluctuation of the prediction curve using NN is more intense than that of the $\mathrm{CC}$ condition. The prediction 
curve using SGAGM and reference curve are basically the same throughout the forecast period. The errors of SoC estimation under different operating conditions are shown in Fig. 6. Clearly, GM $(1,1)$ shows a high error in Fig. 5; thus, GM $(1,1)$ is no longer compared to Fig. 6. The SGAGM can achieve a higher SoC estimation accuracy than $\operatorname{GM}(1,1)$ and NN. The mean absolute error (MAE) for SGAGM is less than $1 \%$, and the maximum error is less than $1.04 \%$. The results indicate that SGAGM has an accurate estimation of SoC and a good robustness against varying temperatures and working conditions. Compared to the performance of $\operatorname{GM}(1,1)$, this is mainly because the SGAGM needs only a limited amount of non-negative data for SoC estimation and updating the input in real time. Besides, the NN requires a lot of data training, leading to poor fault tolerance. Thus, the estimated results have greater fluctuations.

Fig. 5. The comparison of SoC estimation for (a) B0005 at 2A; (b) B0029 at 4A; (c) B0033 at 4A; (d) B0053 at 4A; (e) \#11 at JP1015; (f) \# 11 at NEDC.

Fig. 6. Errors of SoC estimation under different operating conditions: (a) MAE; (b) maximum error

\section{B. Analysis of adjustment coefficient}

Considering the SGAGM model, the value of $\lambda$ was the main elements to influence the prediction accuracy of the model. Moreover, as the GA is utilized to calculate and obtain the value of $\lambda$, the optimal value of $\lambda$ is adjusted continually, as shown in Figs. 7 and 8 . Figs. 7 (a) and 8 (a) show the scatter diagrams to exhibit the distribution intervals of adjustment parameter $\lambda$. The distribution of adjustment parameter $\lambda$ under CC discharge is denser than that of adjustment parameter $\lambda$ under dynamic loading condition. In addition to the scatter diagrams, the $95 \%$ confidence intervals (CI) were also calculated. The adjustment parameter $\lambda$ under $\mathrm{CC}$ discharge is distributed in the area of $[0.4937,0.5023]$. However, the adjustment parameter $\lambda$ under JP1015 is distributed in the range [0.3450 0.6642]. The $95 \%$ CIs also indicate that the adjustment parameter $\lambda$ under JP1015 underwent more dramatic changes than the adjustable parameter $\lambda$ under CC discharge. Figs. 7 (b) and 8 (b) show the histograms; they show an accurate graphical representation of the distribution of adjustment parameter $\lambda$. Both the histograms are symmetric and unimodal, i.e., the adjustment parameter $\lambda$ is generally a type of normal distribution. However, the fluctuation of $\lambda$ under JP1015 is more volatile than $\mathrm{CC}$ discharge. Therefore, the adjustment parameter $\lambda$ dynamically changes during the discharging. In addition, the fiercer the change in discharge current, the higher the change in $\lambda$. Nevertheless, the adjustment parameter $\lambda$ was maintained around 0.5 , even though it changed dynamically during the discharging. This also explains that the adjustment parameter $\lambda$ could correct the estimation results, so that the entire system is more in line with the real situation. Furthermore, the estimation accuracy of GM was also improved using the adjustment parameter $\lambda$. In addition, the dynamically changing values of $\lambda$ indicate the effectiveness of the optimal effects for GAs. As a result, the proposed SGAGM can achieve a high SoC estimation accuracy.

To further illustrate the optimal effect of $\lambda$ to improve the accuracy of SoC estimation, the MRE, degree of grey relation, and post-error ratio were determined. The predicted performance qualities of GM $(1,1)$ and SGAGM were evaluated and compared in Table IV. SGAGM $(8,1,1)$ showed a better performance than GM $(1,1)$ at different discharging rates, ambient temperatures, and dynamic working conditions. The MRE for SGAGM $(8,1,1)$ could win four orders of magnitude back compared to GM $(1,1)$, indicating that SGAGM $(8,1,1)$ can achieve the best predicted result. Because the Sliding Mechanism was used in $\operatorname{SGAGM}(8,1,1)$, the newest information was utilized in modeling; thus, the predicted accuracy was significantly improved compared to GM $(1,1)$. Notably, the accuracy of these two models were affected by the discharge rate as shown in Fig. 5 and Table IV. Experiments under the same temperature but different discharge rates show that the higher the discharge rate, the lower the predicted accuracy. The larger the discharge rates, the lower the predicted accuracy. This is probably because of the more intensive side effect of battery. The nonlinearity of battery is strong, but the SGAGM can achieve the highest accuracy. The mean square error ratio $C$ is the ratio of error variance and predicted data variance. The smaller the value of $C$, the more stable the prediction. The $C$ of SGAGM was smaller than GM $(1,1)$ either in a dynamic or constant test, i.e., the predicted result of SGAGM is more stable. The degree of grey relation $\gamma$ was used to describe the related degree of approximation of predicted data (the estimated SoC) and raw data (the reference SoC). Clearly, the related degree of SGAGM is higher than GM $(1,1)$. This indicates that the estimated result of SGAGM is closer to the reference SoC, i.e., the SGAGM achieved a higher predicted accuracy. Considering the effect of the optimal value of $\lambda$, both the predicted accuracy model show that the SGAGM has a good rejection capability for a predicted model with good dynamic performance and robustness.

Fig. 7. Parameters $\lambda$ of SGAGM change in the CC discharge test of B0005: (a) scatter diagram of parameters $\lambda$ and (b) histogram with a distribution fit

Fig. 8. Parameters $\lambda$ of SGAGM change in the JP1015 discharge test of \# 11: (a) scatter diagram of parameters $\lambda$ and (b) column diagram with a distribution fit

TABLE IV

EVALUATION RESULT USING GM $(1,1)$ AND SGAGM

\section{CONCLUSIONS AND FUTURE WORK}

SoC, a critical index for BMS, is essential for EV performance and reliability. In this paper, to accurately estimate the SoC, the GM-based sliding window mechanism and GA are proposed. The main conclusions are as follows:

(1) The GM is proposed to estimate SoC. To further improve the estimation accuracy of GM, the sliding window mechanism was used to dynamically update the model parameters of GM $(1,1)$ and GAs to optimize the adjustment coefficient of GM (1, 1), combining GM to build SGAGM. The estimation result of GM $(1,1)$ was also selected to compare with SGAGM, and three standard criteria were selected to evaluate and determine the accuracy of grey prediction models.

(2) A battery test-setup was built, and a series of experiments under different discharge rates, conditions, and temperatures were conducted to evaluate the performance of the proposed method. 
(3) A high accuracy of SoC estimation was achieved based on SGAGM. The estimation accuracy of traditional NN was also compared with the proposed SGAGM. The results show that the accuracy of the SoC estimation using SGAGM is the best under different discharge conditions, including the dynamic condition of NEDC and JP1015. Thus, the proposed SGAGM is a viable method for SoC prediction and suitable for EV application.

In the follow-up study, experiments would be carried out using some latest modular hardware from NI (National Instruments) CompactRIO, and the experiment platform would be expanded to achieve an energy storage system. In addition, a combined SoC and SoH online estimation method over the lifespan of LIBs is also being considered.

\section{REFERENCES}

[1] Y. Hu, X. Song, W. Cao, and B. Ji, "New SR Drive With Integrated Charging Capacity for Plug-In Hybrid Electric Vehicles (PHEVs)," IEEE Transactions on Industrial Electronics, vol. 61, pp. 5722-5731, 2014.

[2] L. Lu, X. Han, J. Li, J. Hua, and M. Ouyang, "A review on the key issues for lithium-ion battery management in electric vehicles," Journal of Power Sources, vol. 226, pp. 272-288, 2013.

[3] S. M. Rezvanizaniani, Z. Liu, Y. Chen, and J. Lee, "Review and recent advances in battery health monitoring and prognostics technologies for electric vehicle (EV) safety and mobility," Journal of Power Sources, vol. 256, pp. 110-124, 2014.

[4] C. Campestrini, M. F. Horsche, I. Zilberman, T. Heil, T. Zimmermann, and A. Jossen, "Validation and benchmark methods for battery management system functionalities: State of charge estimation algorithms," Journal of Energy Storage, vol. 7, pp. 38-51, 2016.

[5] F. Sun, R. Xiong and H. He, "Estimation of state-of-charge and state-of-power capability of lithium-ion battery considering varying health conditions," Journal of Power Sources, vol. 259, pp. 166-176, 2014.

[6] C. Hu, B. D. Youn and J. Chung, "A multiscale framework with extended Kalman filter for lithium-ion battery SOC and capacity estimation," Applied Energy, vol. 92, pp. 694-704, 2012.

[7] L. Zhong, C. Zhang, Y. He, and Z. Chen, "A method for the estimation of the battery pack state of charge based on in-pack cells uniformity analysis," Applied Energy, vol. 113, pp. 558-564, 2014.

[8] J. Li, J. K. Barillas, C. Guenther, and M. A. Danzer, "A comparative study of state of charge estimation algorithms for LiFePO 4 batteries used in electric vehicles," Journal of Power Sources, vol. 230, pp. 244-250, 2013.

[9] M. A. Awadallah and B. Venkatesh, "Accuracy improvement of SOC estimation in lithium-ion batteries," Journal of Energy Storage, vol. 6, pp. 95-104, 2016.

[10] J. K. Barillas, J. Li, C. Günther, and M. A. Danzer, "A comparative study and validation of state estimation algorithms for Li-ion batteries in battery management systems," Applied Energy, vol. 155, pp. 455-462, 2015.

[11] W. Waag and D. U. Sauer, "Adaptive estimation of the electromotive force of the lithium-ion battery after current interruption for an accurate state-of-charge and capacity determination," Applied Energy, vol. 111, pp. 416-427, 2013.

[12] H. Aung, K. S. Low and T. G. Shu, "State-of-Charge Estimation of Lithium-Ion Battery Using Square Root Spherical Unscented Kalman Filter (Sqrt-UKFST) in Nanosatellite," IEEE Transactions on Power Electronics, vol. 30, pp. 4774-4783, 2015.

[13] J. Li, J. K. Barillas, C. Guenther, and M. A. Danzer, "A comparative study of state of charge estimation algorithms for $\mathrm{LiFePO} 4$ batteries used in electric vehicles," Journal of Power Sources, vol. 230, pp. 244-250, 2013.

[14] Y. Xing, W. He, M. Pecht, and K. L. Tsui, "State of charge estimation of lithium-ion batteries using the open-circuit voltage at various ambient temperatures," Applied Energy, vol. 113, pp. 106-115, 2014.

[15] L. W. Kang, X. Zhao and J. Ma, "A new neural network model for the state-of-charge estimation in the battery degradation process," Applied Energy, vol. 121, pp. 20-27, 2014.

[16] W. Waag, C. Fleischer and D. U. Sauer, "Critical review of the methods for monitoring of lithium-ion batteries in electric and hybrid vehicles," Journal of Power Sources, vol. 258, pp. 321-339, 2014.

[17] J. N. Hu, J. J. Hu, H. B. Lin, X. P. Li, C. L. Jiang, X. H. Qiu, and W. S. Li, "State-of-charge estimation for battery management system using optimized support vector machine for regression," Journal of Power Sources, vol. 269, pp. 682-693, 2014.

[18] T. Hansen and C. J. Wang, "Support vector based battery state of charge estimator," Journal of Power Sources, vol. 141, pp. 351-358, 2005.

[19] Y. Wang, H. Fang, Z. Sahinoglu, T. Wada, and S. Hara, "Adaptive Estimation of the State of Charge for Lithium-Ion Batteries: Nonlinear Geometric Observer Approach," IEEE Transactions on Control Systems Technology, vol. 23, pp. 948-962, 2015.

[20] H. Chaoui, N. Golbon, I. Hmouz, R. Souissi, and S. Tahar, "Lyapunov-Based Adaptive State of Charge and State of Health Estimation for Lithium-Ion Batteries," IEEE Transactions on Industrial Electronics, vol. 62, pp. 1610-1618, 2015.

[21] E. Kayacan, B. Ulutas and O. Kaynak, Grey system theory-based models in time series prediction: Pergamon Press, Inc., 2010.

[22] T. Y. Pai, H. M. Lo, T. J. Wan, L. Chen, P. S. Hung, H. H. Lo, W. J. Lai, and H. Y. Lee, "Predicting air pollutant emissions from a medical incinerator using grey model and neural network," Applied Mathematical Modelling, vol. 39, pp. 1513-1525, 2015.

[23] L. Wu, S. Liu, Z. Fang, and H. Xu, "Properties of the GM(1,1) with fractional order accumulation," Applied Mathematics \& Computation, vol. 252, pp. 287-293, 2015.

[24] S. S. Chen, J. H. Xu and Y. Fan, "Evaluating the effect of coal mine safety supervision system policy in China's coal mining industry: A two-phase analysis," Resources Policy, vol. 46, pp. 12-21, 2015.

[25] C. I. Chen and S. J. Huang, "The necessary and sufficient condition for GM(1,1) grey prediction model," Applied Mathematics \& Computation, vol. 219, pp. 6152-6162, 2013.

[26] P. P. Xiong, Y. G. Dang, T. X. Yao, and Z. X. Wang, "Optimal modeling and forecasting of the energy consumption and production in China," Energy, vol. 77, pp. 623-634, 2014.

[27] G. D. Li, C. H. Wang, S. Masuda, and M. Nagai, "A research on short term load forecasting problem applying improved grey dynamic model," International Journal of Electrical Power \& Energy Systems, vol. 33, pp. 809-816, 2011.

[28] L. Chen, B. Tian, W. Lin, and B. Ji, "Analysis and prediction of the discharge characteristics of the lithium - ion battery based on the Grey system theory," Power Electronics Iet, vol. 8, pp. 2361-2369, 2015.

[29] L. Chen, W. Lin, J. Li, B. Tian, and H. Pan, "Prediction of lithium-ion battery capacity with metabolic grey model," Energy, vol. 106, pp. 662-672, 2016.

[30] D. E. Goldberg, "Genetic Algorithms in Search, Optimization and Machine Learning," vol. xiii, pp. 2104 - 2116, 1989.

[31] S. F. Zhang, S. Y. Liu and R. H. Zhai, "An extended GRA method for MCDM with interval-valued triangular fuzzy assessments and unknown weights 尔," Computers \& Industrial Engineering, vol. 61, pp. 1336-1341, 2011.

[32] B. Saha and G. Kai, "Uncertainty Management for Diagnostics and Prognostics of Batteries using Bayesian Techniques," in Aerospace Conference, 2008, pp. 1-8.

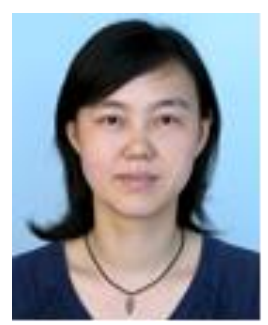

Lin Chen received the M.S. degree in computer applied technology from Guangxi University, Nanning, China, in 2004. She received the Ph.D. degree in biomedical engineering from Huazhong University of Science and Technology, Wuhan, China, in 2008.

From 2011, she was a Professor of College of Mechanical Engineering, Guangxi University. She has hosted and participated in as many as 10 national or regional fund projects. She has published more than 50 technical papers. More than 20 patents have been granted. Her research interests mainly include automation and control, robotics, digital signal detection and processing, AC servo drives, battery management technology and other related research work. 


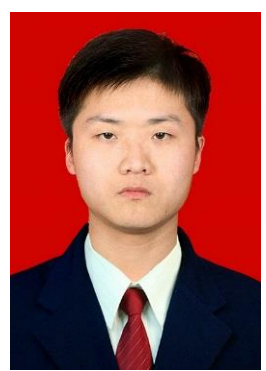

Zhengzheng Wang is currently pursuing the M.S. degree in power machinery and engineering at Guangxi University, Nanning, China. His research mainly focuses on the battery management system in electric vehicles.

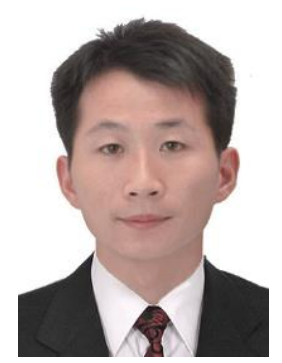

Zhiqiang Lü is currently pursuing the M.S. degree in mechatronic engineering at Guangxi University, Nanning, China. His research mainly focuses on the battery management system in electric vehicles.

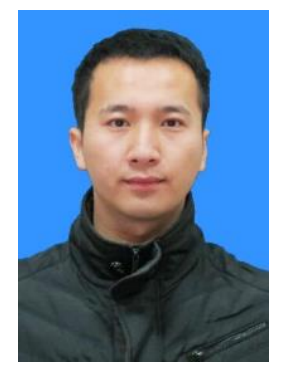

Junzi Li received the M.S. degree in mechanical engineering from Guangxi University, Nanning, China, in 2017.

He currently works in BYD Company Limited, Shenzhen, China, mainly engaged in electric vehicle manufacturing and battery management system research and development.

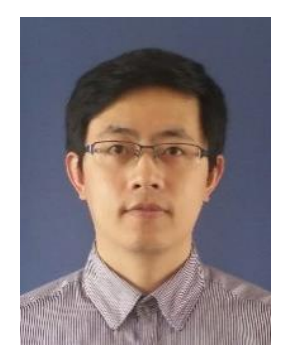

Bing Ji (M'13) received the M.Sc. and Ph.D. degrees in electrical and electronic engineering from Newcastle University, U.K., in 2007 and 2012 respectively.

From 2012, he worked on the electrical powertrain and battery management systems of electric vehicles as a power electronics engineer with a UK low-emission vehicle company. He has worked on different projects as a Postdoctoral Researcher at Newcastle University between 2013 and 2015, looking into accurate power loss measurement and health management for power electronics. He joined the University of Leicester as a Lecturer of Electrical Power Engineering in 2015. His research interests include reliability of power semiconductor devices, batteries and converters, function integration of gate drivers, electro-thermal modeling, thermal management and high power-density converter integration for electric vehicle applications.

Dr. Ji is also a member of the IET.

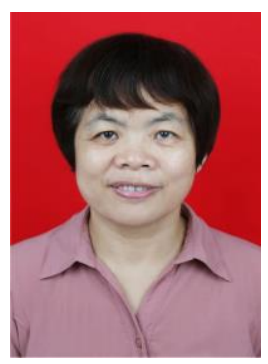

Haiyan Wei received the Ph.D. degree in vehicle application engineering from Jiangsu University, Jiangsu, China, in 2010.

From 2004, she was a associate Professor of College of Mechanical Engineering, Guangxi University. She has hosted and participated in as many as 9 regional fund projects. She has published more than 20 technical papers. More than 20 patents have been granted. His research interests include New energy vehicle power plant, vehicle energy saving and emission control, vehicle power matching and other related research work.

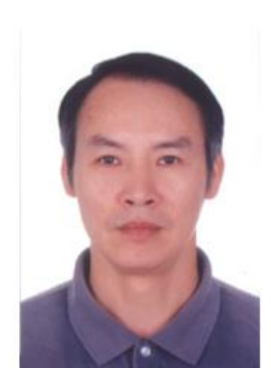

Haihong Pan received the Ph.D. degree in mechanical engineering from Huazhong University of Science and Technology, Wuhan, in 2007.

From 2008, he was a Professor of College of Mechanical Engineering, Guangxi University. He has hosted and participated in as many as 16 national or regional fund projects. He has published more than 60 technical papers. More than 50 patents have been granted. His research interests include electromechanical control theory and method of complex electromechanical system, all digital networked AC servo drive motor control theory and technology and other related research work. 


\section{Figures}

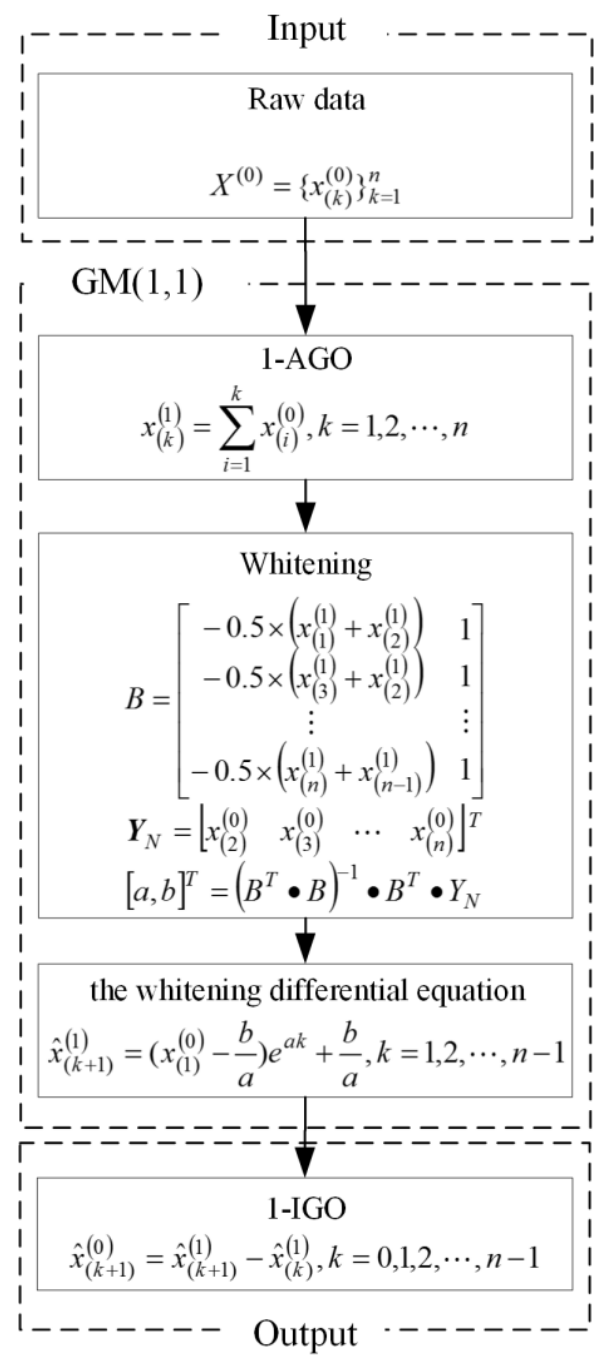

Fig.1. Implementation schematic of GM $(1,1)$ 


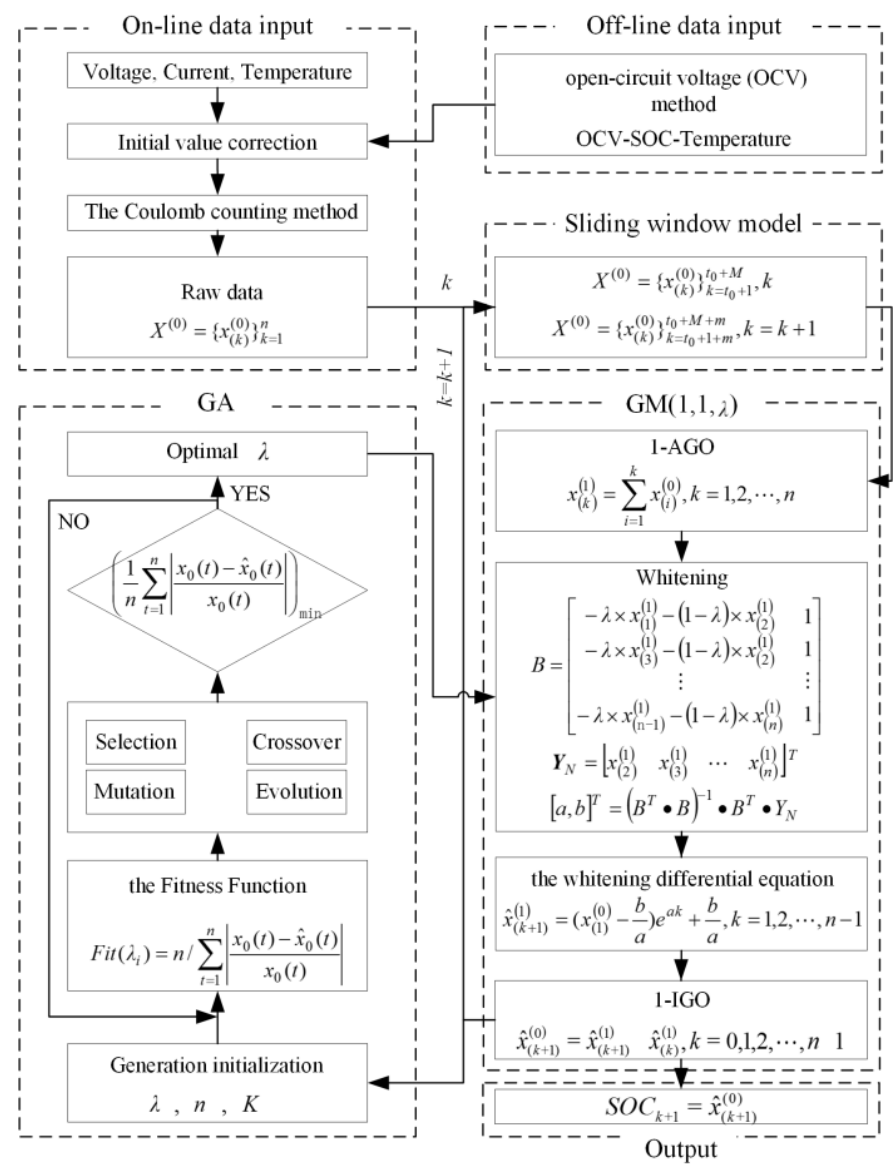

Fig. 2. Implementation schematic of SGAGM for SoC estimation 


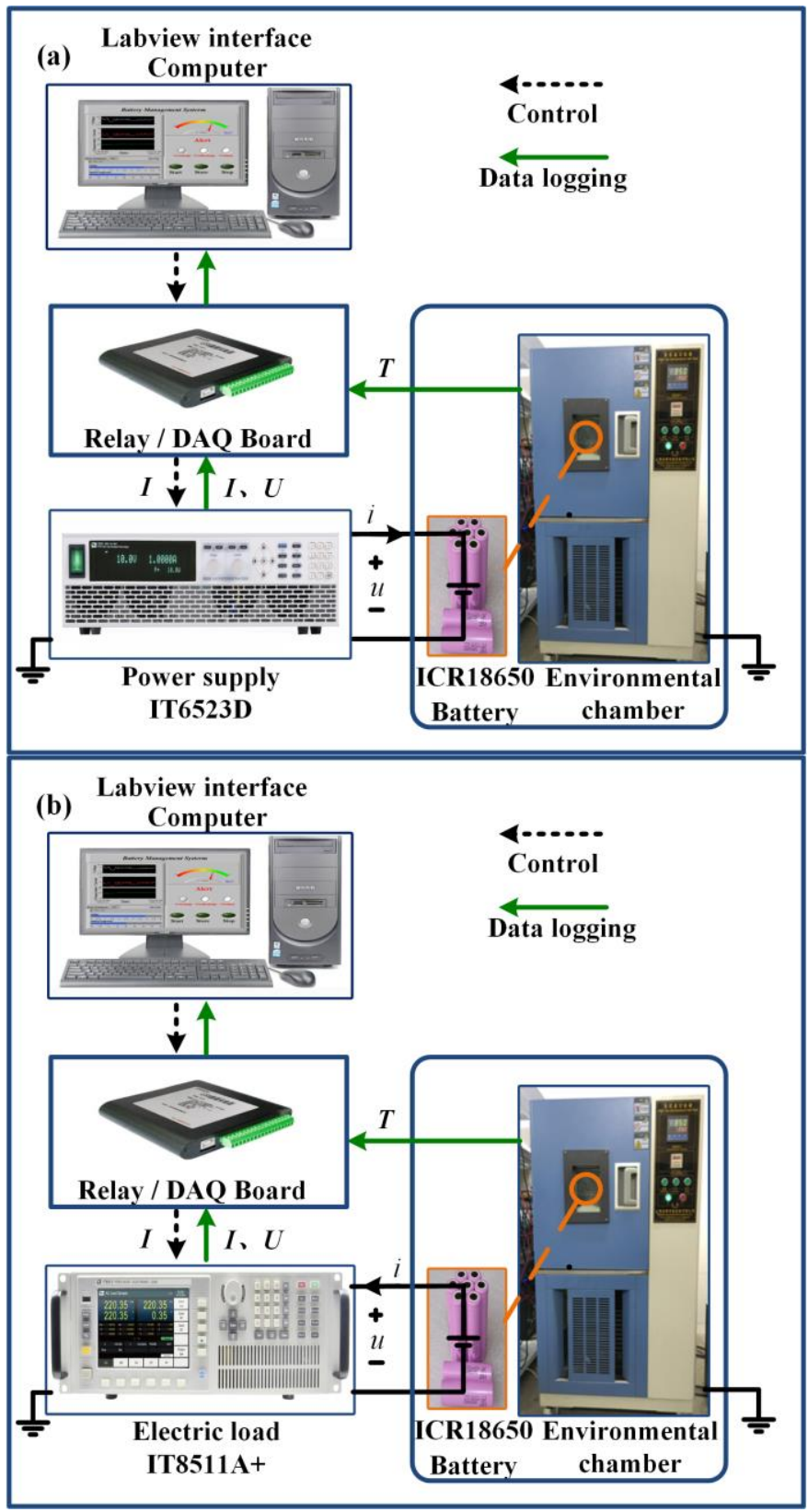

Fig. 3. Physical experimental platform: (a) charging process of battery, (b) discharging process of battery 

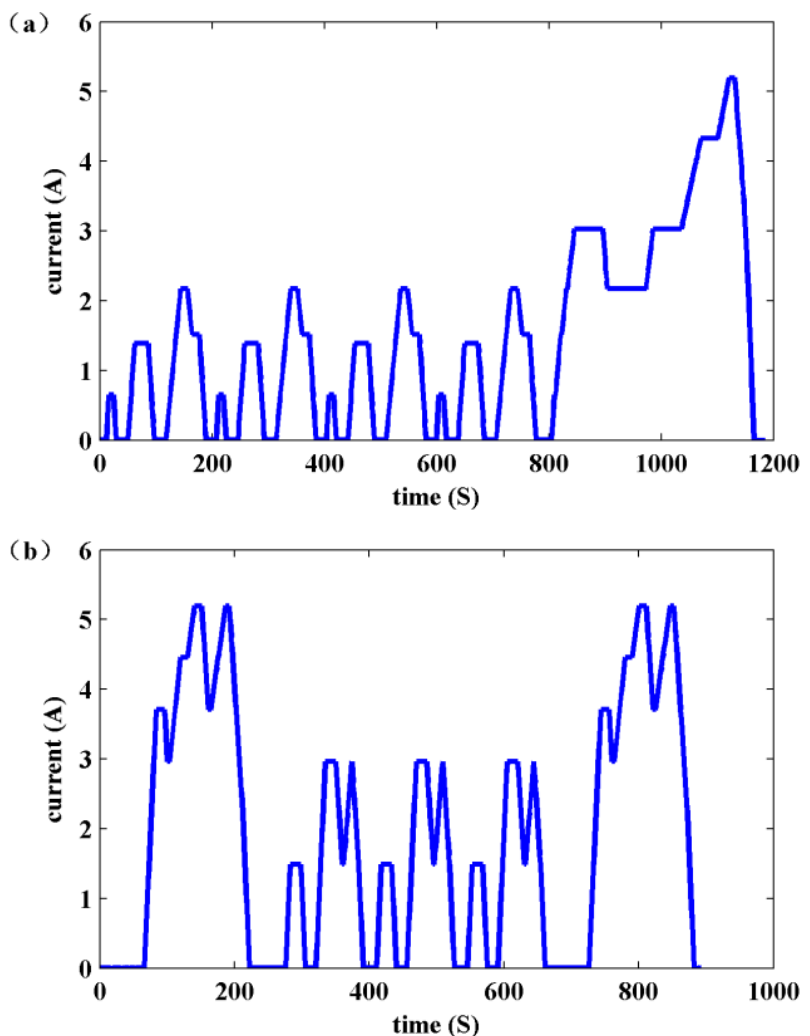

Fig. 4. Measured cell response in the dynamic condition test. (a) New European Driving Cycle. (b) Japanese 10.15 Model Driving Schedule 

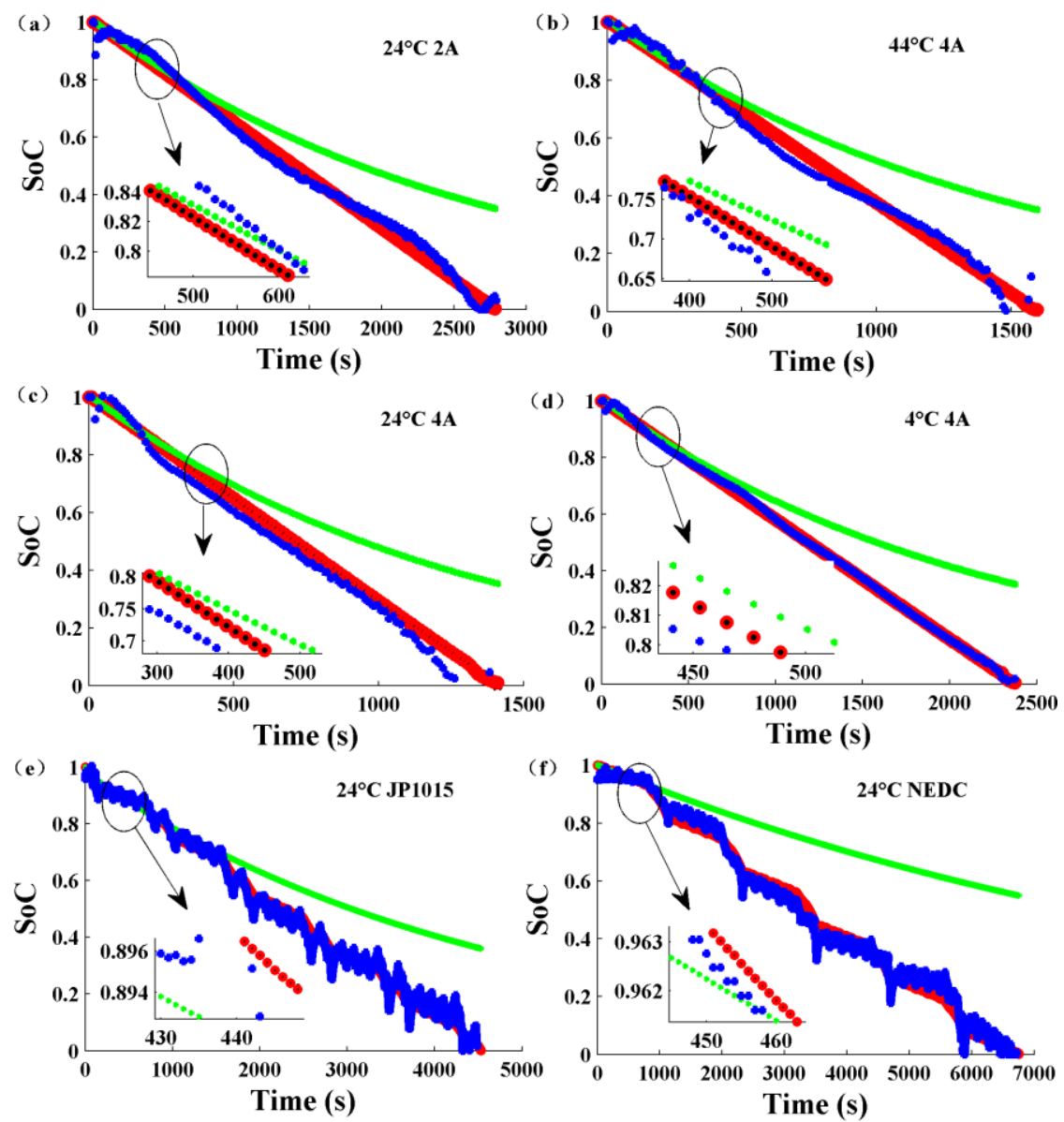

\begin{tabular}{llllll}
$\times$ & Reference SoC & $\operatorname{SGAGM}(8,1,1)$ & $\mathrm{GM}(1,1)$ & $\mathrm{NN}$ \\
\hline
\end{tabular}

Fig. 5. The comparison of SoC estimation for (a) B0005 at 2A; (b) B0029 at 4A; (c) B0033 at 4A; (d) B0053 at 4A; (e) \#11 at JP1015; (f) \# 11 at NEDC. 

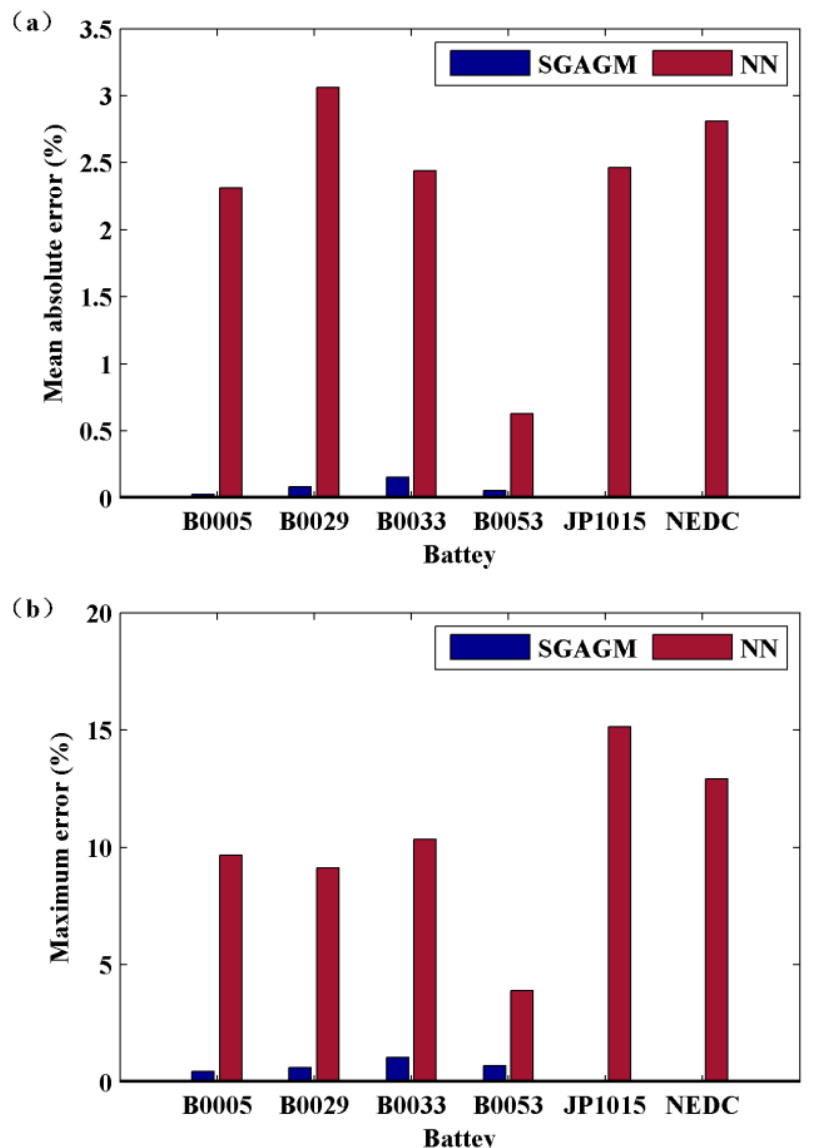

Fig. 6. Errors of SoC estimation under different operating conditions: (a) MAE; (b) maximum error
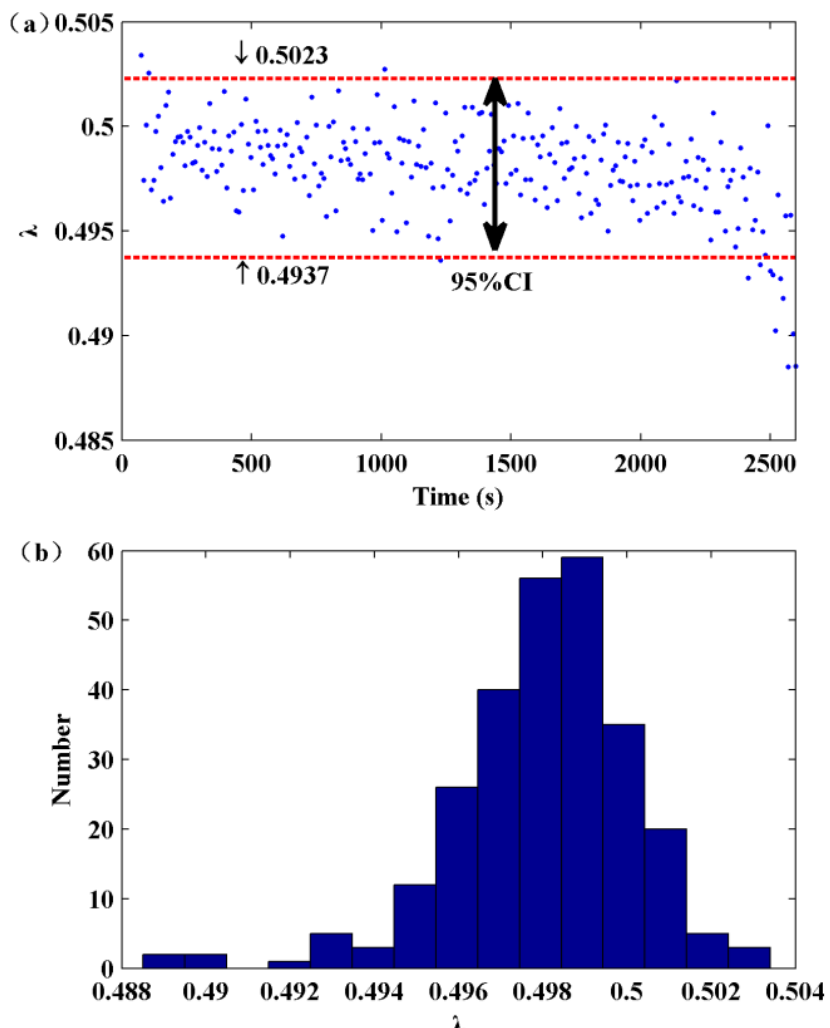

Fig. 7. Parameters $\lambda$ of SGAGM change in the CC discharge test of B0005: (a) scatter diagram of parameters $\lambda$ and (b) histogram with a distribution fit 

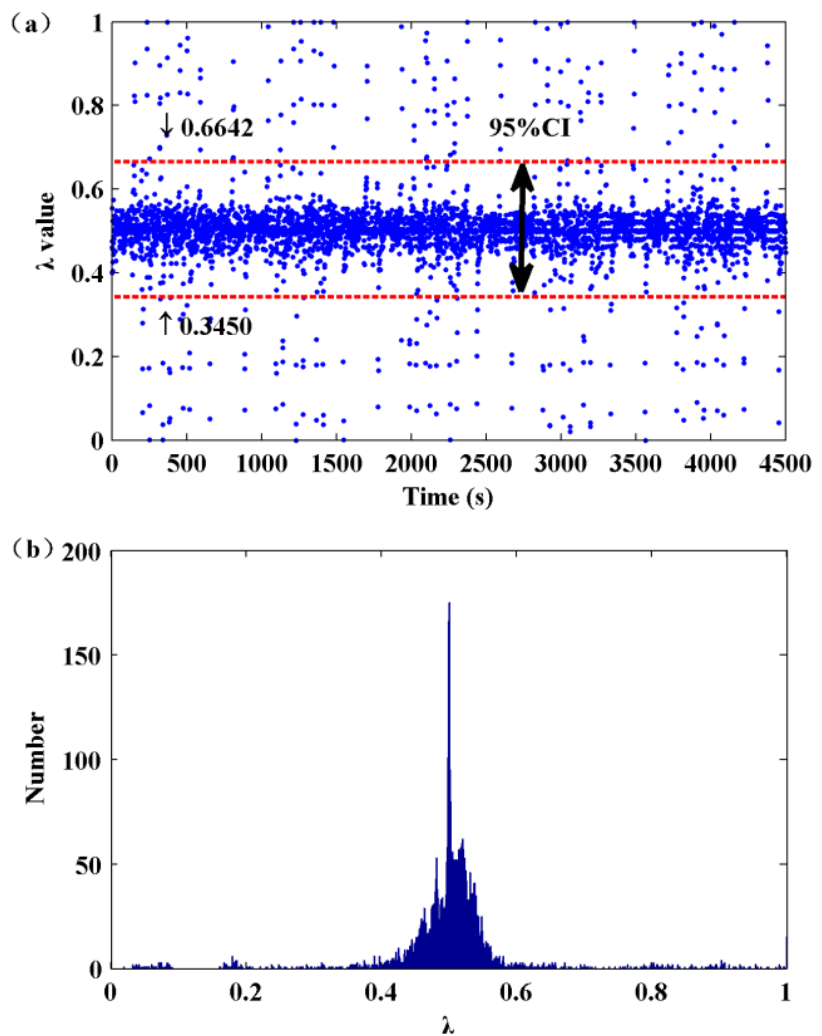

Fig. 8. Parameters $\lambda$ of SGAGM change in the JP1015 discharge test of \# 11: (a) scatter diagram of parameters $\lambda$ and (b) column diagram with a distribution fit 
Nomenclatures

\begin{tabular}{|llll|}
\hline SoC & State-of-charge & EV & Electric vehicle \\
PHEV & Plug-in hybrid electric vehicle & LIB & Lithium-ion battery \\
PEV & Plug-in electric vehicle & BMS & Battery management system \\
OCV & Open-circuit voltage & ECM & Equivalent circuit model \\
EKF & Extended Kalman filter & NN & Neural network \\
SVM & Support vector machine & PI & Proportional integral \\
GM & Grey Model & GA & Genetic Algorithm \\
SGAGM & Sliding genetic algorithms grey model & AGO & Accumulated generating operation \\
MRE & Mean Relative Error & IAGO & Inverse accumulated generating operation \\
GRA & Grey Relational Analysis & CC & Constant current \\
CV & Constant voltage & NEDC & New European Driving Cycle \\
COV & Cut off voltage & JP1015 & Japanese 10.15 Model Driving Schedule \\
CI & confidence intervals & & \\
\hline
\end{tabular}

\section{Tables}

TABLE I

MAJOR EQUIPMENT PARAMETERS

\begin{tabular}{lll}
\hline \hline \multicolumn{1}{c}{ Device name } & Maximum Voltage $(\mathrm{V})$ & Maximum current $(\mathrm{A})$ \\
\hline ITECH IT6523D & $160 \mathrm{~V} \pm 0.2 \%$ full scale & $120 \mathrm{~A} \pm 0.2 \%$ full scale \\
IT8511 A+ & $120 \mathrm{~V} \pm 0.0 .05 \%$ full scale & $30 \mathrm{~A} \pm 0.1 \%$ full scale \\
\hline \hline
\end{tabular}

TABLE II

A COMPARISON OF THE SPECIFICATIONS OF TWO TYPES OF BATTERIES

\begin{tabular}{|c|c|c|}
\hline Specifications $\quad$ Battery & $\begin{array}{c}\text { Type } 1 \\
(18650 \text { Gen } 2)\end{array}$ & $\begin{array}{c}\text { Type } 2 \\
\text { (Samsung ICR18650) }\end{array}$ \\
\hline Rated capacity (Ah) & 2.0 & 2.6 \\
\hline Nominal voltage (V) & l & 3.7 \\
\hline Charge cut-off Voltage & 4.2 & 4.2 \\
\hline Charging Method & $\mathrm{CC}-\mathrm{CV}$ & $\mathrm{CC}-\mathrm{CV}$ \\
\hline Charging Current & 1.5 & 1.3 \\
\hline
\end{tabular}


TABLE III

LIST OF EXPERIMENTAL BATTERIES

\begin{tabular}{|c|c|c|c|c|}
\hline Conditions & Temperature & $4^{\circ} \mathrm{C}$ & $24{ }^{\circ} \mathrm{C}$ & $44^{\circ} \mathrm{C}$ \\
\hline $2 \mathrm{~A}$ & & 1 & B0005 & I \\
\hline $4 \mathrm{~A}$ & & B0053 & B0033 & B0029 \\
\hline NEDC & & / & $\# 11$ & 1 \\
\hline JP1015 & & I & \#11 & I \\
\hline
\end{tabular}

TABLE IV

EVALUATION RESULT USING GM $(1,1)$ AND SGAGM

\begin{tabular}{|c|c|c|c|}
\hline Battery number & $\begin{array}{l}\text { Evaluation } \\
\text { parameters }\end{array}$ & $\operatorname{GM}(1,1)$ & SGAGM \\
\hline \multirow{3}{*}{ B0005 } & $\alpha$ & 0.1323 & $2.799 \times 10^{-4}$ \\
\hline & $C$ & 0.0014 & 0.0016 \\
\hline & $\gamma_{0}$ & 0.9985 & 0.6742 \\
\hline \multirow{3}{*}{ B0029 } & $\alpha$ & 0.1372 & $8.502 \times 10^{-4}$ \\
\hline & $C$ & 0.0035 & 0.0096 \\
\hline & $\gamma_{0}$ & 0.9955 & 0.6742 \\
\hline \multirow{3}{*}{ B0033 } & $\alpha$ & 0.1335 & $1.7 \times 10^{-3}$ \\
\hline & C & 0.0071 & 0.0098 \\
\hline & $\gamma_{0}$ & 0.9913 & 0.6730 \\
\hline \multirow{3}{*}{ B0053 } & $\alpha$ & 0.1321 & $5.7288 \times 10^{-4}$ \\
\hline & $C$ & 0.0029 & 0.0098 \\
\hline & $\gamma_{0}$ & 0.9969 & 0.6752 \\
\hline \multirow{3}{*}{ \#11(JP1015) } & $\alpha$ & 0.1377 & $5.7888 \times 10^{-5}$ \\
\hline & $C$ & 0.3175 & $2.912 \times 10^{-4}$ \\
\hline & $\gamma_{0}$ & 0.6652 & 0.9997 \\
\hline \multirow{3}{*}{ \#11(NEDC) } & $\alpha$ & 0.2792 & $3.3814 \times 10^{-5}$ \\
\hline & $C$ & 0.0844 & $1.5423 \times 10^{-4}$ \\
\hline & $\gamma_{0}$ & 0.5897 & 0.9999 \\
\hline
\end{tabular}

